\title{
Left Adrenal Gland Analysis in Lung Cancer Patients Using the Endobronchial Ultrasound Scope: A Feasibility Trial
}

\author{
Laurence M.M.J. Crombag Jouke T. Annema \\ Department of Pulmonology, Academic Medical Centre, University of Amsterdam, Amsterdam, The Netherlands
}

\section{Key Words}

Lung cancer · Endobronchial ultrasound · Esophageal

ultrasound · Left adrenal gland

\begin{abstract}
Background: In lung cancer patients, the adrenal glands are predilection sites for distant metastases. Esophageal endoscopic ultrasound - fine-needle aspiration (EUS-FNA) is a minimally invasive and accurate method for left adrenal gland (LAG) analysis but requires a conventional gastrointestinal echoendoscope. Complete endobronchial and esophageal mediastinal nodal staging can be achieved by just a single endobronchial ultrasound (EBUS) scope, introducing it into the esophagus (EUS-B) following the endobronchial procedure. Whether the LAG can also be assessed with the EBUS scope is unknown. Objectives: The aim of the study was to investigate the feasibility of identifying the LAG with the EBUS scope. Methods: We conducted a retrospective analysis of lung cancer patients who underwent EBUS and EUS-B for mediastinal staging and LAG assessment between January 2013 and May 2015. Results: A total of 143 patients with (suspected) lung cancer were investigated by the combination of EBUS and EUS-B. In 68 of the 80 patients (85\%) in whom an attempt was made to identify the LAG, it was feasible to transgastrically detect the LAG with the EBUS scope. In 9 patients with endosonographic signs of malignant involvement, diagnostic transgastric FNAs were obtained in
\end{abstract}

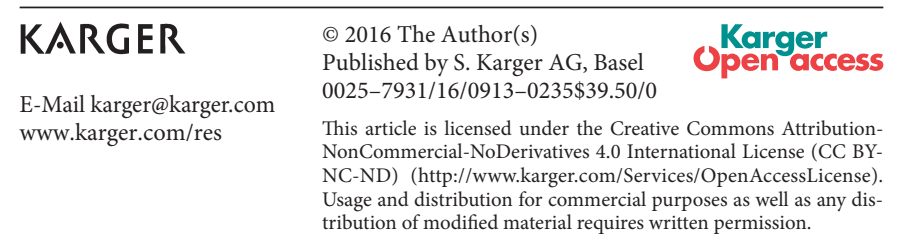

Laurence M.M.J. Crombag

Department of Pulmonology, F5-144, Academic Medical Centre University of Amsterdam, Meibergdreef 9

NL-1105 AZ Amsterdam (The Netherlands)

E-Mail l.m.crombag@amc.nl

\section{Introduction}

Distant metastases have a significant impact on the prognosis and treatment of lung cancer patients. The adrenal glands are a predilection site for lung cancer metastases $[1,2]$. However, even in lung cancer patients, an enlarged adrenal gland is more likely to be benign than malignant [3]. Therefore, additional analysis is needed for accurate staging. Various imaging and sampling techniques are available. A normal adrenal gland consists of a body (size $\leq 8-10 \mathrm{~mm}$ in width) and a lateral and medial limb ( $\leq 5 \mathrm{~mm}$ in width). CT density of an adrenal gland in Hounsfield units on a non-contrast-enhanced CT can be helpful to discriminate a benign from a malignant lesion but is imperfect to rule out malignancy [4]. Fluorodeoxyglucose-positron emission tomography-CT (FDG-

all. In the 12 patients (15\%) in whom the LAG was not detected, the contact between the ultrasound transducer and not a limiting factor. Conclusions: The EBUS scope allows identification of the LAG in the vast majority of lung cancer patients. Implication: In patients with (suspected) lung cancer, in addition to complete hilar and mediastinal staging, LAG assessment using just a single EBUS scope also seems (c) 2016 The Author(s) Published by S. Karger AG, Basel ribution of modified material requires written permission. 
PET-CT) has a high accuracy (a sensitivity of $94 \%$ and a specificity of $85 \%$ ) for adrenal metastases in patients with lung cancer [5]. However, suspicious adrenal glands at FDG-PET-CT can be false positive [6], and therefore, (tissue) verification of FDG-PET avid adrenal glands is essential to ensure accurate staging and appropriate therapy. Traditionally, adrenal masses have been sampled by (CT- or ultrasound-guided) percutaneous biopsy. The reported sensitivity and negative predictive value of adrenal biopsy are 73.3 and 60\%, respectively [7]. The overall reported complication rate for percutaneous adrenal biopsies is $8.4 \%$ (hemorrhage, pneumothorax, pancreatitis and needle-tract metastases) [8]. Nowadays, sampling of the left adrenal gland (LAG) is commonly performed by endoscopic ultrasound - fine needle aspiration (EUSFNA), using a conventional gastrointestinal (GI) echo endoscope. EUS-FNA is a highly accurate method for LAG analysis [9-11] and has a sensitivity for the detection of LAG metastases in lung cancer patients of at least $86 \%$ [12]. EUS-FNA of the LAG has a superior safety profile over percutaneous biopsies as needle insertion is performed under real-time ultrasound guidance and with the transgastric approach, no other organs except the stomach wall is traversed to access the LAG. Complications of EUS-guided FNA of the adrenal glands are rare (adrenal hemorrhage has been described [13]).

Current guidelines regarding mediastinal nodal staging in lung cancer patients advise endosonography in case tissue verification is indicated [14-17]. By combining an endobronchial ultrasound (EBUS) with an EUS(-B) procedure, complete hilar and mediastinal staging can be achieved [18]. Increasing evidence shows that the combined endobronchial and esophageal staging can be performed using just the EBUS scope, introducing it into the esophagus following the endobronchial nodal assessment [19]. This complete staging procedure can be done using a single EBUS scope in one setting and requiring only 1 endoscopist [20-22]. This single-scope approach has several logistical and practical advantages and is obviously cost-effective compared to a dual scope approach.

A recently published small $(n=6)$ case series in selected lung cancer patients showed that the LAG can be identified and sampled using an EBUS scope [23]. However, to date, the feasibility and technique of LAG assessment using the EBUS scope is unknown. Therefore, we aimed to investigate whether and how the LAG could be identified by EUS-B following mediastinal nodal evaluation.

\section{Material and Methods}

\section{Study Design}

We analyzed the data of the endosonography database of the Academic Medical Centre Amsterdam that were prospectively collected. Criteria for patients to be included in the retrospective study were (1) proven or suspected lung cancer, (2) mediastinal nodal assessment by EBUS and EUS-B, and (3) attempt to detect the LAG using the EBUS scope (i.e. EUS-B). The endoscopic procedures were performed in the Academic Medical Centre Amsterdam by 3 experienced chest physicians who are specifically trained in EBUS, EUS, and EUS-B for the diagnosis and staging of lung cancer, including LAG analysis. In our hospital, the combination of EBUS and EUS(-B) in lung cancer patients with an indication for mediastinal nodal staging is performed according to the guideline from June 2015 [18]. EUS(-B) is performed according to the EUS assessment tool (EUSAT) that includes systematic mediastinal nodal staging, including the identification of the liver, coeliac trunk, and the LAG [24].

\section{LAG Assessment by EUS-B (Use of the EBUS Scope)}

In order to identify the LAG, a structured protocol was followed to systematically investigate the abdominal region of interest using the EUSAT [24]. EUSAT is a validated assessment tool specifically developed for lung cancer patients that aims to systematically assess the mediastinal and upper abdominal region of interest. Initially, the liver was visualized from the cardia (fig. 1a). By turning the EBUS scope clockwise from this position, the abdominal aorta and coeliac trunk were identified (fig. 1b). By turning the EBUS scope gently in the caudal direction, the left kidney and LAG were transgastrically identified (fig. 1c). In the case of a sonographic appearance of possible malignant involvement (enlarged corpus or limb, loss of the normal seagull configuration or presence of a mass), samples were taken under real-time ultrasound control (fig. 2b). Prior to sampling, color Doppler was used to identify the blood vessels. EUS-B-FNA was performed using 22- or 25-gauge needles. For LAG sampling, a new separate needle was used. At least 2 LAG samples were taken, and the aspirates were processed for both cytological smears (Giemsa and/or Papanicolaou stains) and cell block analysis. Rapid on-site evaluation was performed in all cases.

\section{Sedation and Equipment}

Conscious sedation by midazolam $(2.5-5 \mathrm{mg})$ i.v. or propofol i.v. was used. EUS-B assessment of the LAG was performed with an ultrasound bronchoscope with a linear scanning transducer (Pentax EB-1970UK; Pentax Medical, Hamburg, Germany) with patients positioned in the supine position during the investigation. A dedicated ultrasound processor was used for imaging processing with Doppler flow imaging for the detection of blood vessels and real-time ultrasound-guided sampling (Hi Vision Preirus, model EZU-MT28-S1; Hitachi Medical Corporation, Reeuwijk, The Netherlands). The patients were observed for $1.5 \mathrm{~h}$ after the procedure. They were instructed to contact the hospital in case of chest discomfort or other complaints.

\section{Statement of Ethics}

Informed consent for endoscopy was obtained in all cases. The Ethics Committee of the Academic Medical Centre Amsterdam has approved LAG analysis in lung cancer patients using the EBUS scope. 


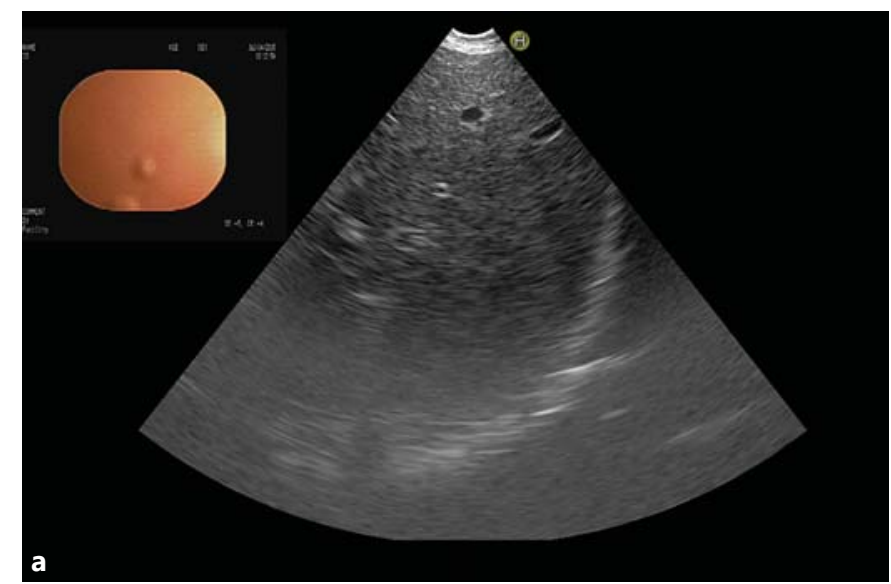

Fig. 1. a In order to find the LAG, a structured three-step approach was followed according to the EUS assessment tool (EUSAT) [24]. This figure shows the liver with the EBUS scope positioned in the stomach. b By turning the EBUS scope clockwise from the liver, the abdominal aorta $(\mathrm{AO})$ and coeliac trunk (CO) are identified. c By turning the EBUS scope gently in the caudal direction, the left kidney (LK) and LAG are transgastrically identified. The LAG has a normal seagull configuration with an adrenal body and 2 limbs.

\section{Results}

During the study period (January 2013-May 2015), 143 patients underwent EBUS and EUS-B for hilar/mediastinal staging of (suspected) lung cancer. In 80 patients (56\%), we attempted to visualize the LAG (fig. 3). In these 80 patients, the final diagnosis was non-small-cell lung cancer (NSCLC, $n=62)$, SCLC $(n=10)$, pulmonary infection $(\mathrm{n}=6)$ and mediastinal metastases of an extrathoracic tumor $(n=2)$. Forty-eight percent of the study population were male, the mean age was 63 years (range $46-$ 84) and the median height was $170 \mathrm{~cm}$ (range 153-198). The majority of patients (85\%) underwent CT-PET, and $15 \%$ of the patients underwent solely CT of the chest and upper abdomen. The CT-PET reports described a normal LAG in 62 patients (78\%), a LAG adenoma in 13 patients (16\%) and a suspected malignant LAG in 5 patients $(6 \%)$.

In 68 of the 80 patients (85\%) in whom we attempted to visualize the LAG, it was feasible to transgastrically identify the LAG using the EBUS scope. In 59 patients (87\%), the LAG had a normal seagull configuration
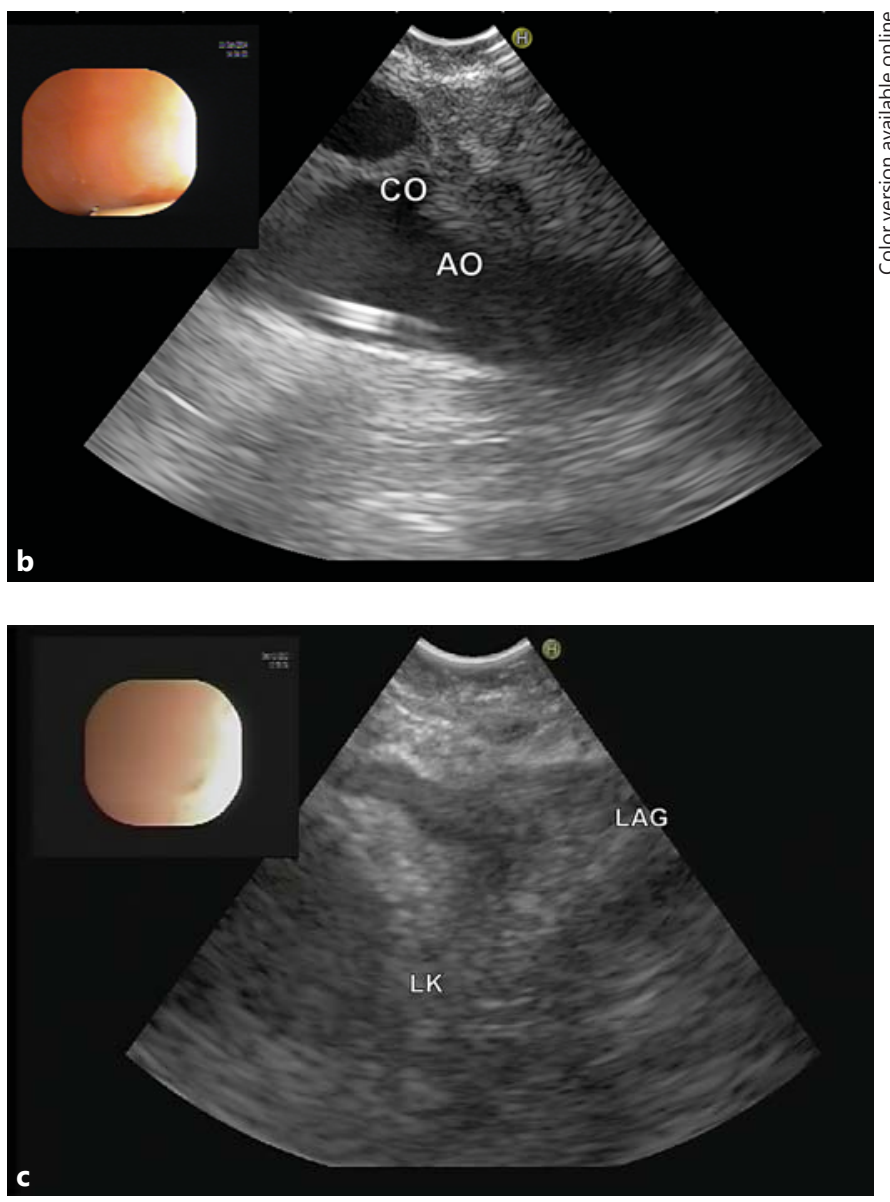

(fig. 1c). In 9 patients, the LAG had an abnormal configuration: 7 patients presented with an enlarged corpus (the short axis ranging from 11 to $25 \mathrm{~mm}$ ) while maintaining the normal seagull configuration, and 2 patients had a LAG mass with loss of the seagull configuration (fig. 2b). In all 9 patients, transgastric FNAs of the LAG (lesions ranging from 11 to $32 \mathrm{~mm}$ ) were performed. The mean number of LAG samples taken was 3.5 (range 2-6). Cytopathologic evaluation yielded benign adrenal tissue in 6 patients, metastases of NSCLC in 2 patients (fig. 2c) and hormonally inactive pheochromocytoma in 1 patient.

In 12 patients, the LAG was not identified due to the poor contact between the ultrasound transducer and stomach wall. Three patients had a hernia diaphragmatica which made it more difficult to maintain firm contact between the gastric wall and ultrasound transducer. The mean height of patients in whom the LAG could not be visualized was $168 \mathrm{~cm}$ (range 153-179). In patients in whom the LAG could be visualized using the EBUS scope, the mean height was $172 \mathrm{~cm}$ (range 154-198). The EUS-B 

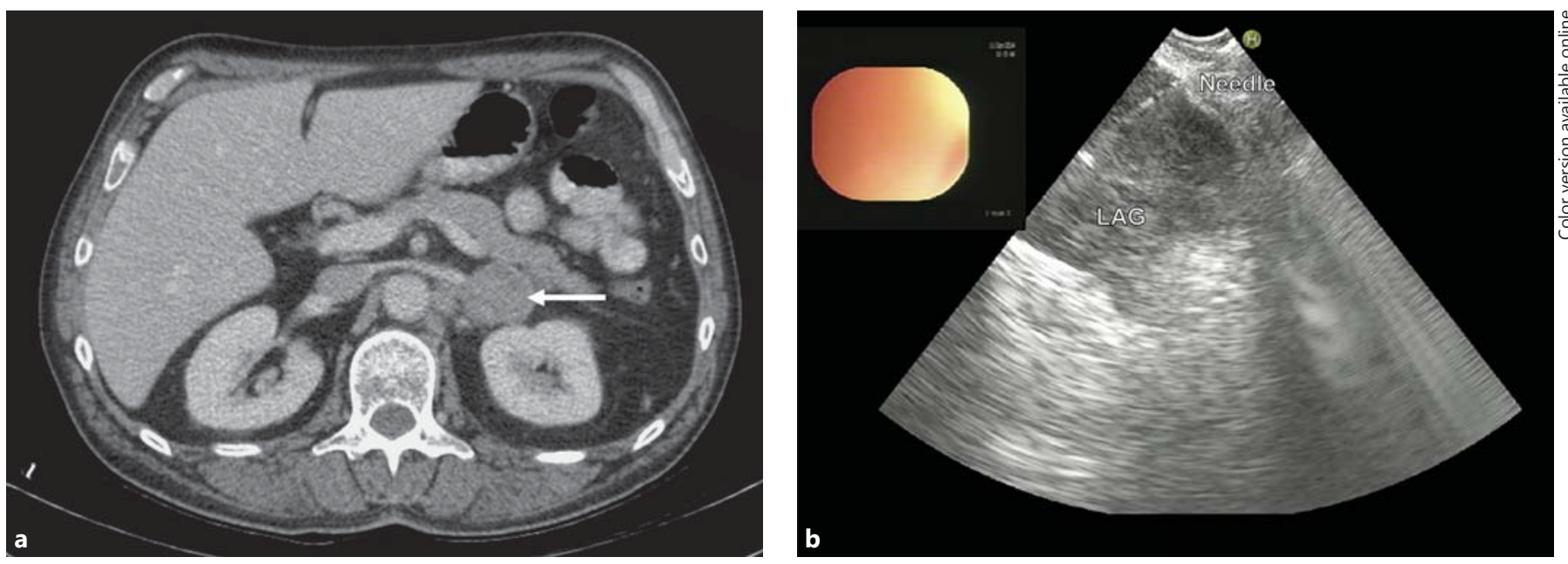

Fig. 2. A 60-year-old smoker with an adenocarcinoma in the right upper lobe, an enlarged subcarinal lymph node and an enlarged LAG. a CT scan of the abdomen, showing an enlarged FDG-avid left adrenal mass (white arrow). b The LAG mass is visualized and transgastrically sampled using the EBUS scope. c Giemsa staining of the cytological aspiration of the LAG mass, revealing metastases of the adenocarcinoma.

procedure had to be aborted prematurely three times due to vomit reflex and imminent aspiration. No other adverse events were noted following the procedure. Transgastric FNA of the LAG was uneventful in all patients.

\section{Discussion}

We found that the LAG could be identified using the EBUS scope (EUS-B) in the vast majority of patients with lung cancer (85\%). Therefore, it seems that in addition to hilar and mediastinal nodal staging, the LAG can also be evaluated with the same EBUS scope in one endoscopy session by a single pulmonologist. In addition to the presumed benefit for patients, the logistical and financial consequences of this strategy are obvious.

Lung cancer is a common malignancy [25]. Accurate staging is important for treatment selection and is a prog-

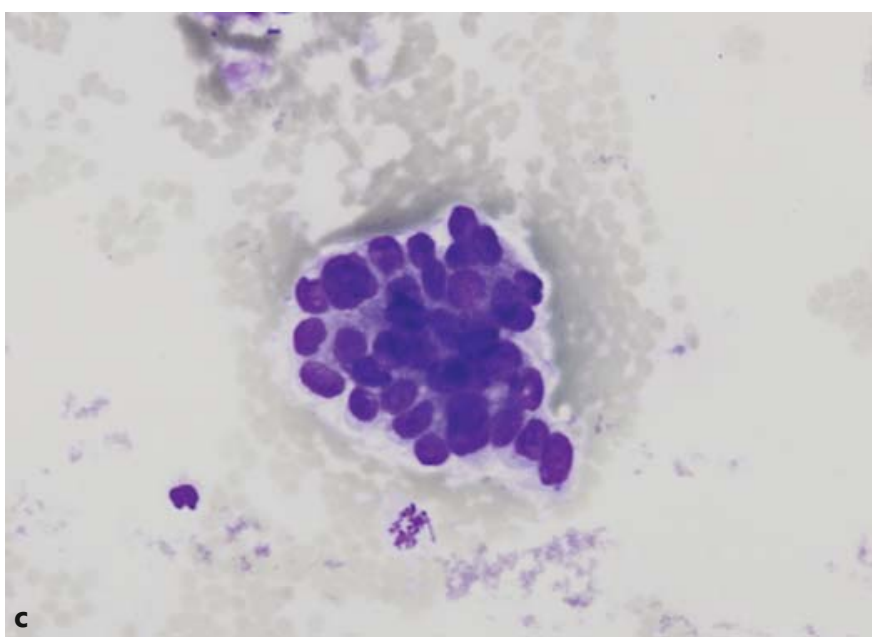

nostic indicator for survival. Fifty-seven percent of the lung cancer patients present with distant metastases (Cancer of the Lung and Bronchus - SEER Stat Fact Sheets), and the adrenal glands are a predilection site. Application of EUS and EUS-FNA to the LAG was initially reported by Chang et al. [9] in 1996. Nowadays, EUS with transgastric FNA is the procedure of choice when tissue confirmation of the LAG in lung cancer patients is indicated [18]. Sampling of the LAG using a conventional EUS scope can be combined with an EBUS scope in a single endoscopy session to ensure a complete endobronchial and esophageal mediastinal nodal staging procedure $[18,26]$. However, using both an EBUS (broncho)scope and an EUS endoscope necessitates the purchase and maintenance of expensive equipment and training in the use of different endoscopes. Moreover, handling of the GI EUS scope is often performed by gastroenterologists, which complicates the logistics. The current data show that following a 


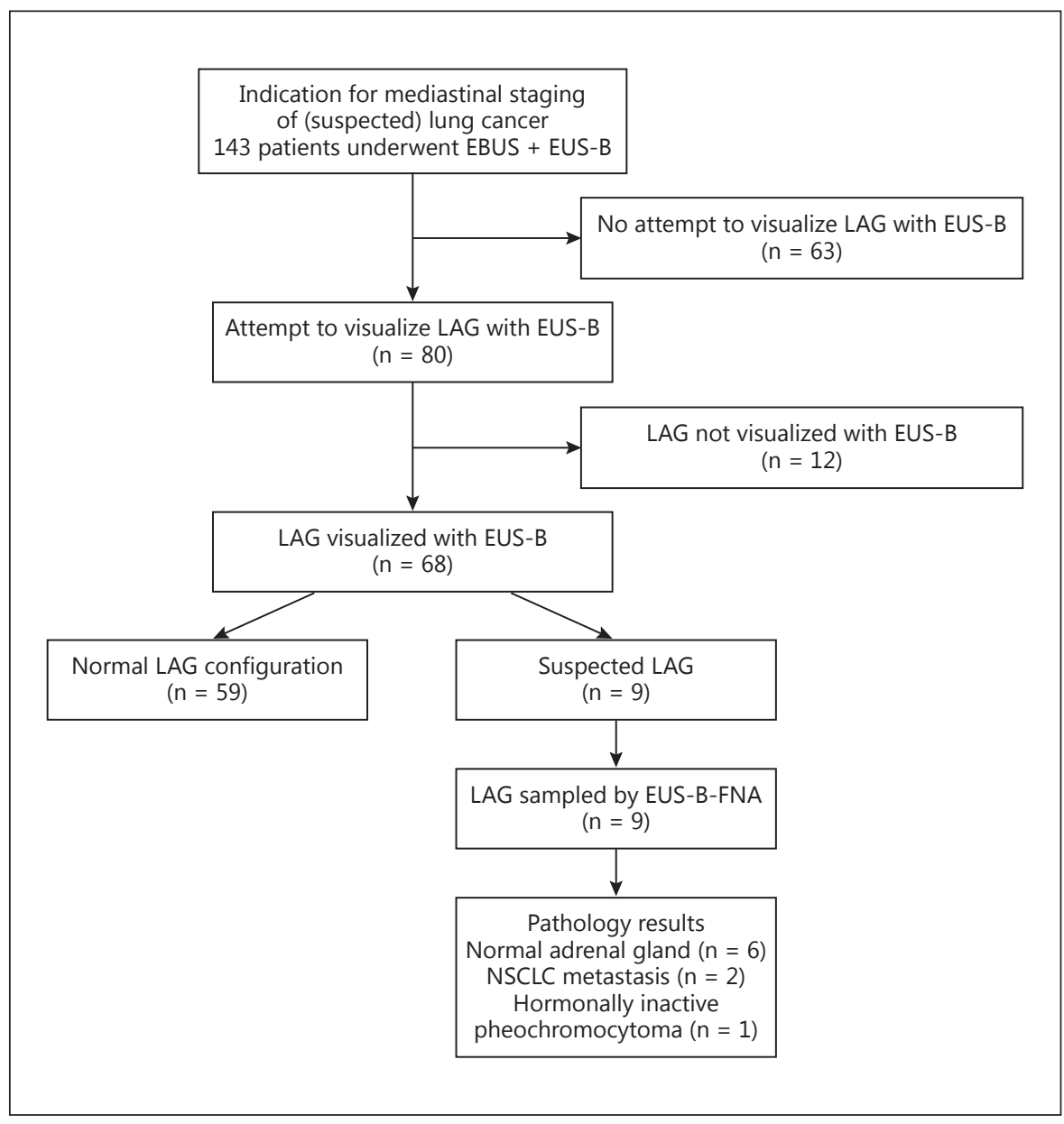

Fig. 3. Flow chart of the study population.

complete mediastinal nodal staging procedure using the EBUS scope, the LAG could be assessed using the same EBUS scope in the same endoscopy session in the vast majority of patients. Therefore, the EBUS scope appears to be an alternative for the conventional EUS assessment of the LAG. In the present study, in $15 \%$ of the patients, the LAG was not found. It needs to be evaluated whether changing the supine position of the patients (the standard position for EBUS) into a left lateral position (the standard position for a conventional EUS examination) leads to further increase in the detection of the LAG.

The conventional GI EUS endoscope is suitable for LAG assessment as the LAG can be identified in $97 \%$ of the cases $[9,10]$. An advantage of the GI EUS scope is its stability when positioned in the stomach compared to the EBUS scope, which is due to its increased tube diameter. This facilitates the contact to the stomach wall and therefore improves the endosonographic assessment of the upper abdomen. Additionally, the conventional echoendoscope has a wider scanning angle, providing the ability to comprehensively image all structures surrounding the region of interest (field of view of approx. $120-180^{\circ} \mathrm{com}-$ pared to $60^{\circ}$ with the EBUS scope). Furthermore, the conventional EUS scope is longer than the EBUS scope, which might be an advantage in tall patients. However, based on the present data, we found no difference in the height of the patients in whom the LAG could and could not be visualized. For assessment of the right adrenal gland, the length of the endoscope will likely be more relevant. In the beginning, it was thought that only the LAG was accessible for endoscopic ultrasound. Recent literature shows that the right adrenal gland is also accessible for transduodenal EUS-guided FNA performed by experienced hands $[16,27]$.

There are several limitations to this study, namely its retrospective design with a possible selection bias, the single-center setting and the limited cases in whom FNA was performed. Subsequent studies - preferably multicenter studies- should include larger cohorts, focus on sampling and include a comparison with the GI EUS scope. 


\section{Conclusion and Implications}

The adrenal glands are predilection sites for lung cancer metastases. Distant metastases have a significant impact on the prognosis and treatment. This study shows that LAG analysis using the EBUS scope is feasible and safe in the vast majority of patients. Since substantial numbers of lung cancer patients have an indication for endosonographic mediastinal lymph node staging, including the need for LAG sampling, a single-scope strat- egy combining complete mediastinal and LAG analysis provides logistical, practical and financial advantages. Most importantly, this strategy simplifies (one single endoscopy session) and speeds up the diagnostic workup of lung cancer patients.

\section{Financial Disclosure and Conflicts of Interest}

The authors declare that they have no conflicts of interest with respect to the content of this paper.

\section{References}

1 Disibio G, French SW: Metastatic patterns of cancers: results from a large autopsy study. Arch Pathol Lab Med 2008;132:931-939.

-2 Quint LE, Tummala S, Brisson LJ, Francis IR, Krupnick AS, Kazerooni EA, et al: Distribution of distant metastases from newly diagnosed non-small cell lung cancer. Ann Thorac Surg 1996;62:246-250.

-3 Abrams HL, Spiro R, Goldstein N: Metastases in carcinoma; analysis of 1,000 autopsied cases. Cancer 1950;3:74-85.

-4 Boland GW, Lee MJ, Gazelle GS, Halpern EF, McNicholas MM, Mueller PR: Characterization of adrenal masses using unenhanced CT: an analysis of the CT literature. AJR Am J Roentgenol 1998;171:201-204.

5 Stone WZ, Wymer DC, Canales BK: fluorodeoxyglucose-positron-emission tomography/ computed tomography imaging for adrenal masses in patients with lung cancer: review and diagnostic algorithm. J Endourol 2014; 28:104-111.

-6 Pieterman RM, van Putten JW, Meuzelaar JJ, Mooyaart EL, Vaalburg W, Koeter GH, et al: Preoperative staging of non-small-cell lung cancer with positron-emission tomography. N Engl J Med 2000;343:254-261.

7 Osman Y, El-Mekresh M, Gomha AM, Mohsen T, Taha N, Hussein N, et al: Percutaneous adrenal biopsy for indeterminate adrenal lesion: complications and diagnostic accuracy. Urol Int 2010;84:315-318.

8 Mody MK, Kazerooni EA, Korobkin M: Percutaneous CT-guided biopsy of adrenal masses: immediate and delayed complications. J Comput Assist Tomogr 1995;19:434-439.

-9 Chang KJ, Erickson RA, Nguyen P: Endoscopic ultrasound (EUS) and EUS-guided fine-needle aspiration of the left adrenal gland. Gastrointest Endosc 1996;44:568-572.

$\checkmark 10$ Eloubeidi MA, Seewald S, Tamhane A, Brand B, Chen VK, Yasuda I, et al: EUS-guided FNA of the left adrenal gland in patients with thoracic or GI malignancies. Gastrointest Endosc 2004;59:627-633.

\section{Eloubeidi MA, Black KR, Tamhane A, Eltoum} IA, Bryant A, Cerfolio RJ: A large single-center experience of EUS-guided FNA of the left and right adrenal glands: diagnostic utility and impact on patient management. Gastrointest Endosc 2010;71:745-753.

-12 Schuurbiers OC, Tournoy KG, Schoppers HJ, Dijkman BG, Timmers HJ, de Geus-Oei LF, et al: EUS-FNA for the detection of left adrenal metastasis in patients with lung cancer. Lung Cancer 2011;73:310-315.

13 Haseganu LE, Diehl DL: Left adrenal gland hemorrhage as a complication of EUS-FNA. Gastrointest Endosc 2009;69:e51-e52.

14 De LP, Dooms C, Kuzdzal J, Lardinois D, Passlick B, Rami-Porta R, et al: Revised ESTS guidelines for preoperative mediastinal lymph node staging for non-small-cell lung cancer. Eur J Cardiothorac Surg 2014;45:787-798.

$\longrightarrow 15$ Silvestri GA, Gonzalez AV, Jantz MA, Margolis ML, Gould MK, Tanoue LT, et al: Methods for staging non-small cell lung cancer: diagnosis and management of lung cancer, ed 3: American College of Chest Physicians evidence-based clinical practice guidelines. Chest 2013;143(5 suppl):e211S-e250S.

-16 Uemura S, Yasuda I, Kato T, Doi S, Kawaguchi J, Yamauchi T, et al: Preoperative routine evaluation of bilateral adrenal glands by endoscopic ultrasound and fine-needle aspiration in patients with potentially resectable lung cancer. Endoscopy 2013;45:195-201.

17 Vansteenkiste J, De RD, Eberhardt WE, Lim E, Senan S, Felip E, et al: Early and locally advanced non-small-cell lung cancer (NSCLC): ESMO Clinical Practice Guidelines for diagnosis, treatment and follow-up. Ann Oncol 2013;24(Suppl 6):vi89-vi98.

18 Vilmann P, Clementsen PF, Colella S, Siemsen M, De LP, Dumonceau JM, et al: Combined endobronchial and oesophageal endosonography for the diagnosis and staging of lung cancer. European Society of Gastrointestinal Endoscopy (ESGE) Guideline, in cooperation with European Respiratory Society (ERS) and the European Society of Thoracic Surgeons (ESTS). Eur Respir J 2015;46:40-60.
9 Zhang R, Ying K, Shi L, Zhang L, Zhou L: Combined endobronchial and endoscopic ultrasound-guided fine needle aspiration for mediastinal lymph node staging of lung cancer: a meta-analysis. Eur J Cancer 2013;49: 1860-1867.

20 Herth FJ, Krasnik M, Kahn N, Eberhardt R, Ernst A: Combined endoscopic-endobronchial ultrasound-guided fine-needle aspiration of mediastinal lymph nodes through a single bronchoscope in 150 patients with suspected lung cancer. Chest 2010;138:790-794.

21 Hwangbo B, Lee GK, Lee HS, Lim KY, Lee SH, Kim HY, et al: Transbronchial and transesophageal fine-needle aspiration using an ultrasound bronchoscope in mediastinal staging of potentially operable lung cancer. Chest 2010;138:795-802.

-22 Kang HJ, Hwangbo B, Lee GK, Nam BH, Lee HS, Kim MS, et al: EBUS-centred versus EUScentred mediastinal staging in lung cancer: a randomised controlled trial. Thorax 2014;69: 261-268.

23 Meena N, Hulett C, Jeffus S, Bartter T: Left adrenal biopsy using the convex curvilinear ultrasound scope. Respiration 2015;89:57-61.

24 Konge L, Vilmann P, Clementsen P, Annema JT, Ringsted C: Reliable and valid assessment of competence in endoscopic ultrasonography and fine-needle aspiration for mediastinal staging of non-small cell lung cancer. Endoscopy 2012;44:928-933.

25 Jemal A, Bray F, Center MM, Ferlay J, Ward E, Forman D: Global cancer statistics. CA Cancer J Clin 2011;61:69-90.

-26 Annema JT, van Meerbeeck JP, Rintoul RC, Dooms C, Deschepper E, Dekkers OM, et al: Mediastinoscopy vs endosonography for mediastinal nodal staging of lung cancer: a randomized trial. JAMA 2010;304:2245-2252.

27 Eloubeidi MA, Morgan DE, Cerfolio RJ, Eltoum IA: Transduodenal EUS-guided FNA of the right adrenal gland. Gastrointest Endosc 2008;67:522-527. 